\title{
Bronchiolitis obliterans following the ingestion of an Asian shrub leaf
}

The introductory article ${ }^{1}$ identifies a potentially important, previously unrecognised, and most unusual cause of obliterative bronchiolitis - a vegetable product ingested in the hope of controlling weight. It reports a clinical study of high quality which not only alerts us to the respiratory hazards of one particular product of the natural environment, but prompts a timely review of two critical aspects of evolving clinical practice in respiratory medicine. These comprise the early diagnostic use of high resolution computed tomographic (HRCT) scanning using inspiratory and expiratory images and the immediate use of specific immunosuppressant therapy.

The study more obviously reminds the practising clinician that the lungs can be readily damaged by toxic agents whose portal of entry is the gastrointestinal tract not the airways, and that serendipity is often necessary before such a problem is recognised. While pulmonary toxicity from a variety of medications is well recognised and regulatory mechanisms now greatly limit the chance of important adverse effects in epidemiological terms, the ingestion of unlicensed medications or "fad" foods carries unknown risks and adverse effects are not readily identified. This theme is also discussed in the article on pages S63-7 by Voelkel reviewing pulmonary hypertension in subjects ingesting appetite suppressants. Uncooked leaf extract of Sauropus androgynus (an Asian shrub of the Euphoriaceae family) is, like appetite suppressant drugs, used to control weight though its effectiveness is not proven. Adverse effects are not seen primarily in the pulmonary vasculature but the introductory article provides very suggestive evidence that it may cause an obliterative bronchiolitis.

Obliterative bronchiolitis

Obliterative bronchiolitis (OB) has remained an enigma, explained in part by common delays in diagnosis. 
F urthermore, open lung biopsy - on which until recently the diagnosis has depended - has often been restricted to the most severely affected patients.

The disease is characterised by persistent inflammation of the bronchioli and proliferative occlusion of the lumen with fibroblasts and smooth muscle cells. ${ }^{23}$ In many forms the airway disease extends centrally to In many forms the airway disease extends centrally to
involve bronchi, which causes bronchiectasis. ${ }^{4} \mathrm{H}$ owever, involve bronchi, which causes bronchiectasis. ${ }^{4} \mathrm{H}$ owever,
the alveoli remain free of the disease process unless the alveoli remain free of the disease process unless
there is evidence of organising pneumonia. The latter there is evidence of organising pneumonia. The latter indicates the separate condition of bronchiolitis obliterans and organising pn

a distinct pathogenesis.

Although OB is not commonly recognised in adults, it is frequently diagnosed in childhood following adenovirus infection and there is a suspicion that it may be caused by respiratory syncytial virus in both children and adults. ${ }^{6}$ O ther established causes include autoimmune diseases such as rheumatoid arthritis, and the inhalation of a number of toxic chemicals such as ammonia chlorine, organophosphates, and nitrogen dioxide. There is also an association with smoking, but the full this is not yet clear. The disorder has gained particular prominence over recent years because of its occurrence complicating heart and lung transplantation, and this has led to greater urgency in obtaining diagnostic biopsy tissue and in administering prompt immunosuppressive therapy. As a result, there have been important advances in understanding the pathogenesis of $\mathrm{OB}$ and in modifying its natural history. pathogenes discovery that it may also occur con ccur commonly in populations ingesting the natural products of certain plants will usefully emphasise its importance in the spectrum of environmentally induced disorders of the airways.

\section{Obliterative bronchiolitis attributable to}

\section{Sauropus androgynus}

During a remarkable period of less than six month more than 60 patients who had ingested juice containing uncooked leaf extract of Sauropus androgynus presented to the Veterans $\mathrm{G}$ eneral H ospital-K aohsiung in T aiwan with progressive undue breathlessness. In the study population of 23 patients the breathlessness was severe and they were admitted, but the size of the population ingesting the ex extract from which the initial 60 were drawn (the denominator) is not known. The epidemiological significance of the outbreak described by $L$ ai and colleagues ${ }^{1}$ is consequently unclear, particularly as news media interest stimulated many of the initial presentations.

The 23 patients reported by $L$ ai et $\mathrm{al}^{1}$ were all women and non-smokers, and all had been well previously. Five had bought the leaf extract as a juice from local stores and 18 had used home made preparations. The authors remarked that the extract was commonly uncooked remarked that the extract was commonly uncooked
when consumed, though is more generally used in Taiwan after cooking. Cooking might therefore "detoxify" the relevant inducer of the bronchiolar disease. Significant heat denaturation would, however, reduce the possibility that the pulmonary reaction was a consequence of papaverine within the fresh leaves (about $580 \mathrm{mg} / 100 \mathrm{~g}$ ) since this is resistant to such levels of heat. The leaf extract was apparently consumed in considerable quantities (estimated range $2-21 \mathrm{~kg}$ over 10 weeks, mean $8 \mathrm{~kg}$ ), so other components will merit consideration as the primary inducing agent.

All 23 patients had progressive undue breathlessness and 21 had cough which was usually non-productive.
There was generally tachypnoea with impaired breath sounds, and there were crackles in 17 and wheezes in three. T he mean forced expiratory volume in one second $\left(\mathrm{FEV}_{1}\right)$ was only $26 \%$ of predicted and forced vital capacity (FVC) $51 \%$, and there was no signficant response to a bronchodilator. A raised mean volume but not total lung capacity indicated air trapping but the corrected gas transfer factor for carbon monoxide (T LCO) was said to be normal in 17. The diagnosis rested essentially on the appearances on the HRCT scan which showed patchy low attenuation of the lung parenchyma with a mosaic perfusion pattern in the expiratory images of all patients and in the inspiratory images of 11. All scans additionally showed bronbronchiectasis in the segmental and subsegmental bronchi. he plain radiographs were essentially normal.

Four patients underwent open lung biopsy which revealed the characteristic appearance of $O B$ with a predominance of $T$ lymphocytes. There was no histological or serological evidence of infection, and material from two biopsy specimens gave negative results when culcured for viruses. The authors specul

$\mathrm{N}$ either bronchodilator nor corticosteroid medication had any discernible effect, and there was no spontaneous resolution once ingestion of the leaf extract was discontinued. Severe respiratory impairment was, however, al ready established before the diagnosis was confirmed in any subject. The authors did not report whether there was continued progression after ingestion ceased.

\section{Obliterative bronchiolitis after heart-lung and}

\section{lung transplantation}

Theintroduction of lung transplant surgery has provided an opportunity to study the natural history of an immunologically "driven" form of OB. In the heartlung transplant population followed by the Stanford group the first descriptions of the presentation, physiology, pathology, and natural history of the disease were described. ${ }^{3}$ Breathlessness and coughing from progressive airflow obstruction were the principal findings associated with the characteristic pathology.

N umerous descriptions followed but a detailed analysis of lung transplant recipients ${ }^{8}$ appeared to indicate that the main risk factor for $\mathrm{OB}$ was the frequency of acute rejection in the first three months after surgery. This complication is now the major long term cause of death after lung transplantation and accounts for a significant amount of morbidity. $\mathrm{N}$ ew forms of immunosuppression including inhaled corticosteroids may delay the development of the disease.

The lessons learnt from transplantation include the value of identifying a progressive fall in $\mathrm{FEV}_{1}$ as a characteristic of $O B$, which cannot be improved even with intense immunosuppressive treatment. Another is the nature of the inflammatory process and of the T lymphocytes which appear to orchestrate the fibroproliferative occlusion of the small airways and cause the bronchiectasis. ${ }^{9}$ T his observation lends support for the principle of early diagnosis and the use of enhanced immunosuppressive treatment in the hope of limiting the rate of decline in $\mathrm{FEV}_{1}$.

An obliterative bronchiolitis is also seen after bone An obliterative bronchiolitis is also seen after bone
marrow transplantation, again driven by an immune process, where graft cells injure the host's airways in a "graft versus host" disease. ${ }^{10}$ Augmented immunosuppressive treatment does offer a means of limiting the rate of progress of this form of disease. 


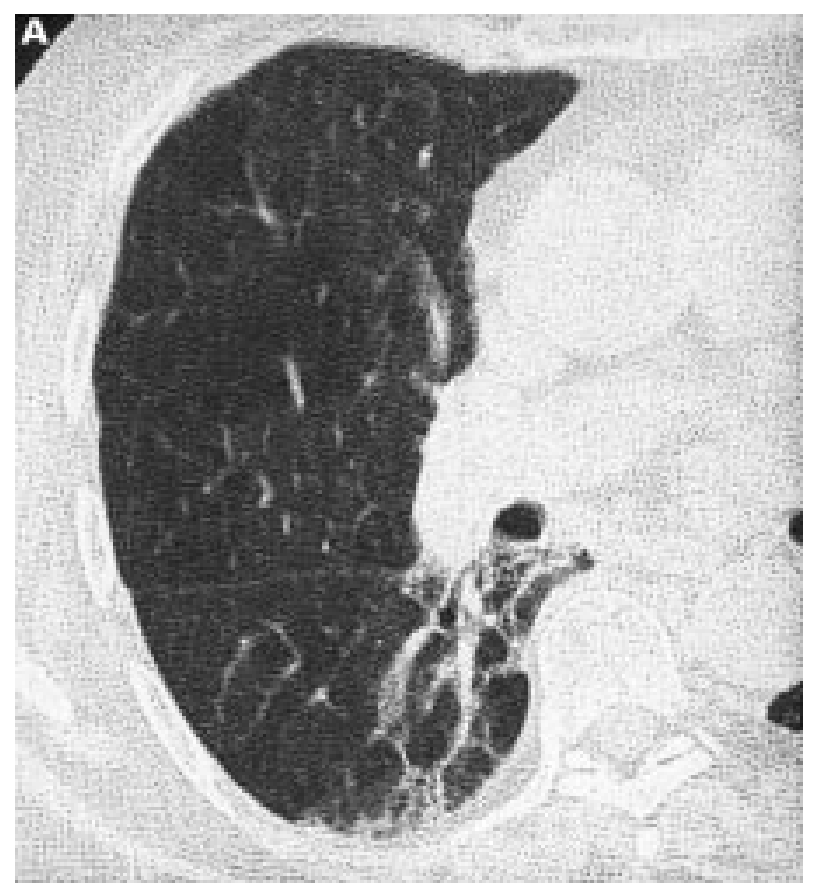

Figure 1 High resolution CT scan in inspiration and expiration. The inspiratory image (A) shows a few fibrotic unremarkable Expiration is inevitably associated with is movement so two expiratory images are shown. In both $B$ (matched for lung level) and C (matched for spinal level) the images show marked variability in attenuation of the lung parenchyma. The more translucent areas identify localsed air trapping and bronchiolar disease. (Courtesy Dr W Simpson).

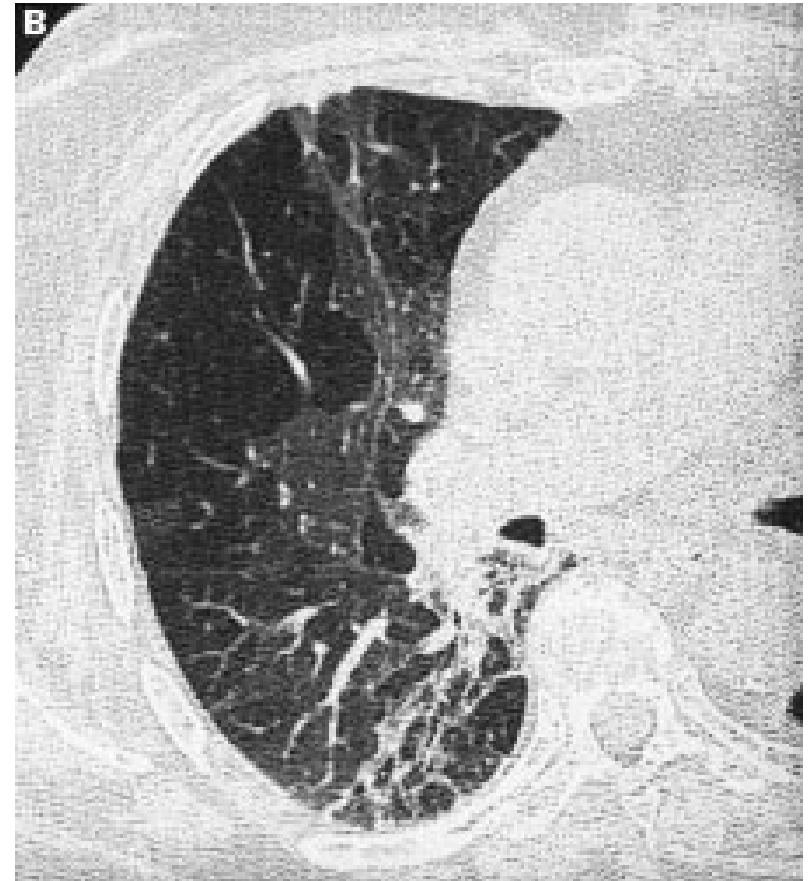

Diagnostic tests for OB

In patients who undergo lung transplantation the observation of a progressive fall in $\mathrm{FEV}_{1}$ without evidence of reversibility provides the first indication of the disease. The TLCo is usually maintained, as is seen in other forms of OB. ${ }^{11}$ The chest radiograph is also normal in appearance, which usefully separates OB from BOOP where irregular, often bilateral, alveolar shadowing is seen.

Progressive irreversible airflow obstruction in transplant patients has proved to be almost always a consequence of $\mathrm{OB}$, so further investigation is not generally indicated. Open lung biopsy, in particular, is no longer required. $F$ or sporadic cases in the population at large, however, there are many potential alternative causes of irreversible airflow obstruction and a progressive decline in $\mathrm{FEV}_{1}$. These include emphysema, asthma, obstructive chronic bronchitis, bronchiectasis, eosinophilic granuloma, and sarcoidosis. Physiological tests can help

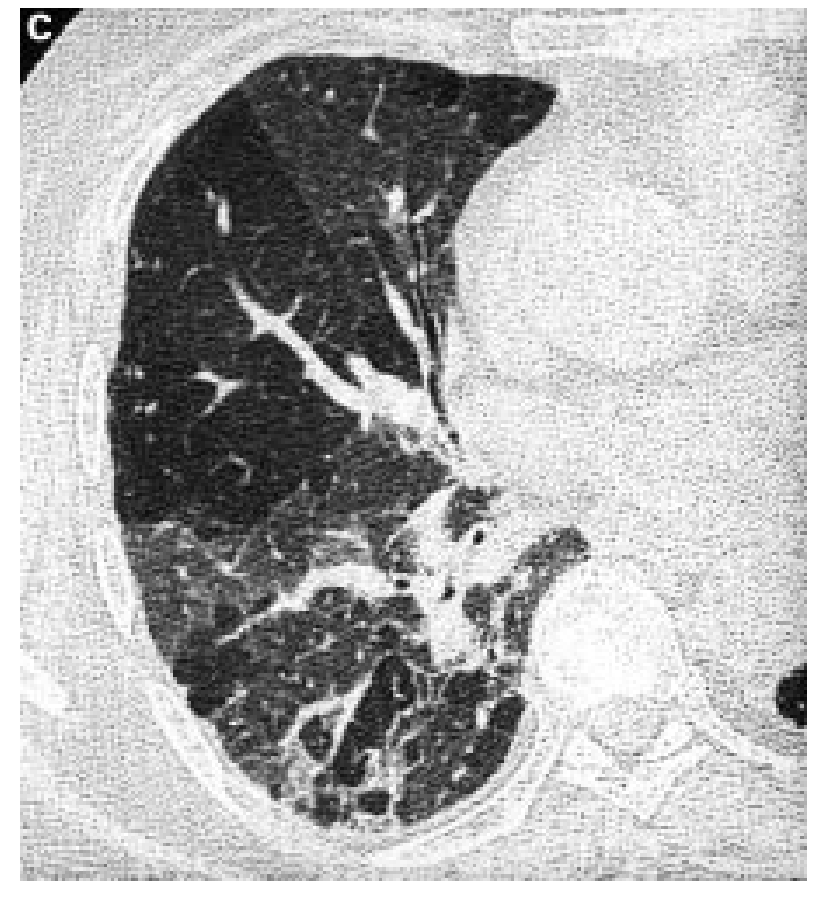

to identify emphysema where the low $\mathrm{FEV}_{1}$ is associated with reduced T LCO and increased total lung capacity, but for most patients alternative measures are usually required.

The diagnosis has depended traditionally on an open lung biopsy, and until the recent introduction of videoassisted thoracoscopy biopsy procedures were reserved for those patients with advanced disease and marked disability - that is, those who have "earned" this ultimate diagnostic test. ${ }^{212}$ Video-assisted thoracoscopy has greatly reduced the risks of lung biopsy whe tissue procedures are required, and biopsy via thoracoscopy procedures are required, and biopsy via thoracoscopy
is indicated when there is a rapid decline in $\mathrm{FEV}_{1}$ despite high dose corticosteroid treatment. Values of $\mathrm{FEV}_{1}$ below one litre often indicate a poor prognosis irrespective of the nature of the underlying disease, and biopsy specimens are commonly taken in these circumstances in non-smoking patients without evidence 
LEARNING POINTS

* Obliterative bronchiolitis (OB) may be induced by the ingestion of an agent present in (or contaminating) the leaf of Sauropus androgynus, an Asian shrub.

* The inducing agent is yet to be identified, but may be susceptible to heat denaturation in cooking.

* OB is best recognised from characteristic CT appearances of patchy low attenuation and a mosaic pattern of perfusion in expiratory images.

* Rapid diagnosis coupled with the identification and elimination of the inducing cause is essential to the prevention of serious respiratory disablement.

* The early use of immunosuppressive therapy may ameliorate the tendency towards progressive disease.

of emphysema. The characteristic pathology is selective involvement of the small airways and proliferative obliteration of the lumina with fibromuscular cells. Inflammatory cells, usually lymphocytes, are seen in the earlier stages. Once there is advanced fibrotic obliteration, little can be offered to the patient in terms of treatment, so there has been a quest for methods of earlier diagnosis.

The introduction of HRCT scanning of the thorax has brought a welcome revolution in clinical practice. has broud of the lung in both full expiration and inspiration has enabled gas trapping and hence bronchiolar disease to be identified reliably. ${ }^{1314}$ Those regions of the lung subserved by narrowed small airways fail to deflate on expiration and retain a low attenuation compared with normal regions. This produces a variegated appearance of low and high attenuation areas. The "patchwork" or "mosaic" appearance on expiration is characteristic pearance on expiration is characteristic of OB an y bronchiectasis to be demonstrated and the exclusion of interstitial diseases, emphysema, and sarcoidosis. ${ }^{15} 16$ I ts use early in the investigation of patients with irreversible airflow obstruction may offer increased hope of successful therapeutic intervention.

\section{Pathogenesis of $O B$}

Little is known of the common pathway towards the obliterative airway process of the different forms of OB. Injury to airway epithelium appears to be a common element. T his may be the first step from which lymphocyte infiltration is initiated. As described in the introductory article $T$ cell lymphocytes are involved, not only in the imm forms as in lung transplantation, but also when ingestion or inhalation of a probable exogenous toxin is involved. Experimental $O B$ has shown that selective suppression of the $T$ cel infiltrate with an agent such as rapamycin "blocks" the subsequent fibromuscular proliferation which is the hallmark of the later stages of the disease.

\section{Treatment of $\mathrm{OB}$}

In lung transplant patients, who are at high risk of developing OB after experiencing three or more rejection episodes in the first three months of transplantation, it has proved possible to prevent $\mathrm{OB}$. This has been achieved by the use of nebulised high dose budesonide a corticosteroid which locally reduces lymphocyte in- filtration whilst avoiding the consequences of high dose oral steroids. T rials are currently underway to study the effect of inhaled steroids in $O B$ from other causes such as graft versus host disease and rheumatoid disease. The same approach might be of value early after toxin ingestion or inhalation injury.

For the patient with advanced disease, where the principal pathology is of progressive fibrous obliteration of the airways, transplantation of the lungs is the main therapeutic option. In these patients, there is no lung transplant is effective. A double lung transplant is required if there is concomitant bronchiectasis. There is no evidence that $\mathrm{OB}$ occurs more frequently after transplantation in patients who have $O B$ as an original diagnosis. Selection of patients for transplantation requires evidence of a poor prognosis, and generally requires an $\mathrm{FEV}_{1}$ below one litre (or less than $30 \%$ predicted), ${ }^{17}$ or dependency on supplemental oxygen.

\section{Conclusions}

The description of an outbreak of OB associated with an "environmental" toxin serves to alert us to the possibility of causes of small airways disease unassociated with cigarette smoking or asthma. The introduction of high speed H RCT scanning to detect gas trapping from small airways disease offers an opportunity for early diagnosis of this devastating disease. From our experience of lung transplantation it now seems possible that, with "early" intervention with specific immunosuppressants such as rapamycin or inhaled high the progressive loss of functioning small airways. It is the progressive loss of functioning small airways. It is
important from the clinical, pathological and therapeutic important from the clinical, pathological an
viewpoints to separate OB from BOOP.

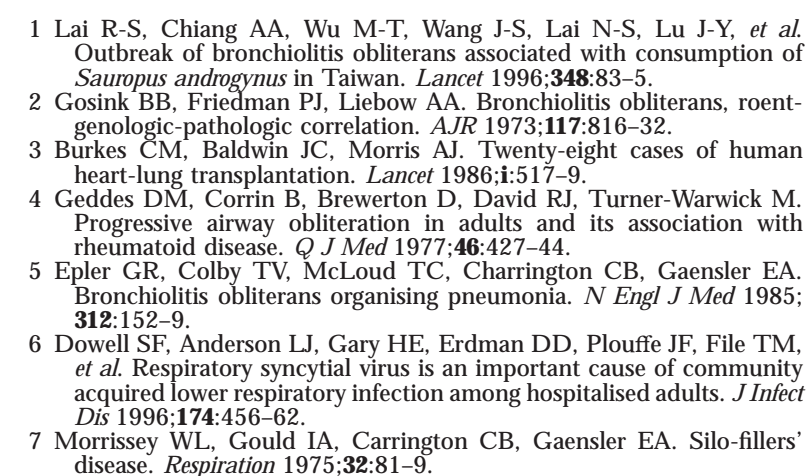


$\mathrm{S} 72$

8 Sharples LD, Tamm M, M CN eil K, Higenbottam TW, Stewart S

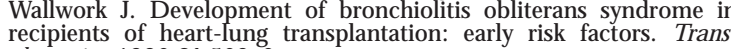
$9 \begin{gathered}\text { plantation 1996;61:560-6. } \\ 9 \text { ertz M I, Henke CA, N akleh RE. Obliterative bronchiolitis after lung }\end{gathered}$ transplantation: a fibroproliferative disorder associated with platelet growth factors. Proc N att A cad Sci 1992;89:10,385-8.
E pler GR. Bronchiolitis obliterans and airways obstruction associated with graft-versus-host disease. Clin C hest $M$ ed 1988;9.551-6s.
w 11 Scott JP, Higenbottam TW, Cieland M B. N Natural history of chronic
rejection in heart-lung transplant recipients. I H eart Transplant 1990; 2 Wright JL, Cagle P, Churg A, Colby TV, M yers J. Diseases of the small

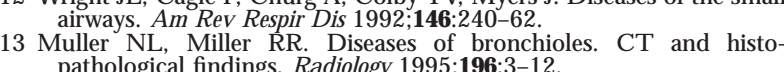

Higenbottam 14 Zerhouni EA, H rold CJ, Brown RH, Wetzel RC, Hirschman CA,
Robotham JL, et al. H High resolution computed tomography - physiologic correlation. I Thorac Imaging 1993;8:265-72.
lany Remy-Jardin M, G iraud F, Remy J, Coplin KC, Gosselin B, D ahemi
A. Importance of ground glass attenuation in chronic diffuseinfiltrative lung disease. Pathologic-CT correlation. Radiology 1993; 189:693-8. M CG uiness G, B eacher JR, H ankin TJ, Garay SM, Rom WN , N ardich
DP. H aemoptysis: prospective high-resolution CT /bronchoscopic correlation. Chest 1994; 105:1155-62.

$17 \mathrm{~K}$ anner $R E$, Renzette $A D$, Stanish WM. Predictions of survival in 18 du Bois RM, G eddes DM. Obliterative bronchiolitis, cryptogenic organising pneumonitis, and bronchiolitis obliterans organising pneu4:774-5. 